\title{
A Regime Switching Model of Schooling Choice as a Job Search Process
}

\author{
Yong Hyun Shin ${ }^{1}$ and Ho-Seok Lee ${ }^{2}$ \\ ${ }^{1}$ Department of Mathematics, Sookmyung Women's University, Seoul 04310, Republic of Korea \\ ${ }^{2}$ Research Institute of Finance \& Risk Management, POSTECH, Pohang 37673, Republic of Korea
}

Correspondence should be addressed to Ho-Seok Lee; kaist.hoseoklee@gmail.com

Received 23 September 2015; Accepted 28 October 2015

Academic Editor: Kiseop Lee

Copyright (C) 2015 Y. H. Shin and H.-S. Lee. This is an open access article distributed under the Creative Commons Attribution License, which permits unrestricted use, distribution, and reproduction in any medium, provided the original work is properly cited.

We propose a regime switching model of schooling choice as a job search process. We adopt a two-state Markov process and the derived coupled Bellman equations are solved by seeking the root of an auxiliary algebraic equation. Some numerical examples are also considered.

\section{Introduction}

In the course of schooling as a job search process, the role of uncertainty that gives rise to an option was studied by Fan [1]. Fan [1] used an analytic formulation, in which the wage offer process $w(t)$ was modeled as the following arithmetic Brownian motion:

$$
d w(t)=g d t+\sigma d B_{t},
$$

where $g$ is a positive constant and $B_{t}$ is a standard Brownian motion. If $Q$ is the schooling preference net of education costs, an individual's objective is to maximize the expected value $V$ with the schooling duration $\tau$ :

$$
V:=\mathbb{E}\left[\int_{0}^{\tau} e^{-r t} Q d t+\int_{\tau}^{\infty} e^{-r t} w(\tau) d t\right],
$$

where $r$ is the constant discount rate and $Q$ was also assumed to be constant in Fan [1]. However, this abstraction may not be able to fully capture reality. Specifically, in an economic recession, the desire to obtain education may rise; for example, graduate program enrollment increases in an economic recession (Fan [1]), but the cost of education, for example, tuition fees, may decline (Binder [2]). In addition, wage offer volatility may have a higher value in an economic recession (Gronau [3]) than in an economic expansion. Therefore, we extend the model by allowing wage offer dynamics and schooling preference (net of education costs) to be modulated by a two-state Markov process. Since an arithmetic Brownian motion allows for a negative wage offer, we utilize a geometric Brownian motion as the wage offer process in this paper.

Our methodology to derive the closed-form solutions is in line with that used in the real option framework with regime switching, such as Dixit and Pindyck [4], Guo et al. [5], and Bensoussan et al. [6].

\section{The Model}

We assume that a risk-neutral individual receives a wage offer $w(t)$, which follows a geometric Brownian motion with coefficients modulated by a two-state Markov process as follows:

$$
d w(t)=\mu_{\Theta_{t}} w(t) d t+\sigma_{\Theta_{t}} w(t) d B_{t}, \quad w(0)=w>0
$$

where $\left(B_{t}\right)_{t \geq 0}$ is a standard Brownian motion under a given probability space $(\Omega, \mathscr{F}, \mathbb{P})$ and $\left(\Theta_{t}\right)_{t \geq 0}$ is a two-state Markov process defined on $(\Omega, \mathscr{F}, \mathbb{P})$ and can take value 1 or 2 . We also assume that $\left(\Theta_{t}\right)_{t \geq 0}$ is independent of $\left(B_{t}\right)_{t \geq 0}$ and has the following generator:

$$
\Lambda=\left(\begin{array}{cc}
-\lambda_{1} & \lambda_{1} \\
\lambda_{2} & -\lambda_{2}
\end{array}\right), \quad \lambda_{1}>0, \lambda_{2}>0 .
$$


For each regime $i,(i=1,2), \mu_{i}$ is a constant drift and $\sigma_{i}$ is a constant volatility. We allow that schooling preference (net of education costs), denoted by $Q_{t}=Q_{\Theta_{t}}$, is also regime switching.

We assume the following as in Fan [1] (see also verifications therein). An individual will stop schooling at time $\tau$ to accept a wage offer, which will be his constant wage throughout the remainder of the individual's infinite lifetime. Further, this stopping process is irreversible. Then an individual's objective is to choose optimally the schooling duration $\tau_{i}$ that maximizes the expected value $V_{i}(w)$, which consists of the present value of lifetime earnings and schooling preference (net of education costs) for each regime $i,(i=1,2)$,

$$
\begin{aligned}
& V_{i}(w):=\max _{\tau_{i}} \mathbb{E}\left[\int_{0}^{\tau_{i}} e^{-r t} Q_{\Theta_{t}} d t\right. \\
& \left.+\int_{\tau_{i}}^{\infty} e^{-r t} w\left(\tau_{i}\right) d t \mid \Theta_{0}=i, w(0)=w\right] \\
& =\max _{\tau_{i}} \mathbb{E}\left[\int_{0}^{\tau_{i}} e^{-r t} Q_{\Theta_{t}} d t+e^{-r \tau_{i}} \frac{w\left(\tau_{i}\right)}{r} \mid \Theta_{0}\right. \\
& =i, w(0)=w]
\end{aligned}
$$

$$
\begin{aligned}
& V_{1}(w)= \begin{cases}A_{3} w^{m_{3}}+A_{4} w^{m_{4}}+\frac{\left(r+\lambda_{2}\right) Q_{1}+\lambda_{1} Q_{2}}{\left(r+\lambda_{1}\right)\left(r+\lambda_{2}\right)-\lambda_{1} \lambda_{2}}, & \text { for } 0<w<\bar{w}_{1}, \\
\frac{w}{r}, & \text { for } w \geq \bar{w}_{1},\end{cases} \\
& V_{2}(w)= \begin{cases}\frac{f_{1}\left(m_{3}\right)}{\lambda_{1}} A_{3} w^{m_{3}}+\frac{f_{1}\left(m_{4}\right)}{\lambda_{1}} A_{4} w^{m_{4}}+\frac{\left(r+\lambda_{1}\right) Q_{2}+\lambda_{2} Q_{1}}{\left(r+\lambda_{1}\right)\left(r+\lambda_{2}\right)-\lambda_{1} \lambda_{2},} & \text { for } 0<w<\bar{w}_{1}, \\
C_{1} w^{n_{1}}+C_{2} w^{n_{2}}+\frac{\lambda_{2}}{r\left(r-\mu_{2}+\lambda_{2}\right)} w+\frac{Q_{2}}{r+\lambda_{2}}, & \text { for } \bar{w}_{1} \leq w<\bar{w}_{2}, \\
\frac{w}{r}, & \text { for } w \geq \bar{w}_{2} .\end{cases}
\end{aligned}
$$

The values of $A_{3}, A_{4}, C_{1}, C_{2}, \bar{w}_{1}$, and $\bar{w}_{2}$ are expanded in the proof.

Proof. From (5), for $0<w<\bar{w}_{1}$, we obtain the following coupled Bellman equations:

$$
\begin{aligned}
& \mathscr{L}_{1} V_{1}(w)-Q_{1}=\lambda_{1} V_{2}(w), \\
& \mathscr{L}_{2} V_{2}(w)-Q_{2}=\lambda_{2} V_{1}(w),
\end{aligned}
$$

where the differential operator $\mathscr{L}_{i}$ is given by

$$
\mathscr{L}_{i} \bullet=-\frac{1}{2} \sigma_{i}^{2} w^{2} \frac{\partial^{2} \bullet}{\partial w^{2}}-\mu_{i} w \frac{\partial \bullet}{\partial w}+\left(r+\lambda_{i}\right) \bullet
$$

where $r$ is the discount rate and $\tau_{i}$ can be considered as an optimal stopping time of education. We denote $w\left(\tau_{1}\right):=\bar{w}_{1}$, $w\left(\tau_{2}\right):=\bar{w}_{2}$ and we only consider the case where

$$
\bar{w}_{1}<\bar{w}_{2}
$$

$\bar{w}_{i}$ is called the reservation wage in labor economics.

Remark 1. For each regime $i,(i=1,2)$, we define the functions $f_{i}(\cdot)$ and $G(\cdot)$ :

$$
\begin{aligned}
& f_{i}(m):=-\frac{1}{2} \sigma_{i}^{2} m(m-1)-\mu_{i} m+\left(r+\lambda_{i}\right), \\
& G(m):=f_{1}(m) f_{2}(m)-\lambda_{1} \lambda_{2} .
\end{aligned}
$$

Let $n_{1}<0$ and $n_{2}>0$ be two real roots of the quadratic equation $f_{2}(n)=0$. We also consider the quartic equation $G(m)=0$, which has four real roots $m_{1}<m_{2}<0<m_{3}<m_{4}$.

Theorem 2. Under assumption (6), for each regime $i,(i=$ $1,2)$, the value function $V_{i}(\cdot)$ is derived as follows: for each regime $i,(i=1,2)$. Thus the solutions to (9) are of the forms

$$
\begin{aligned}
& V_{1}(w)=A_{3} w^{m_{3}}+A_{4} w^{m_{4}}+a, \\
& V_{2}(w)=\frac{f_{1}\left(m_{3}\right)}{\lambda_{1}} A_{3} w^{m_{3}}+\frac{f_{1}\left(m_{4}\right)}{\lambda_{1}} A_{4} w^{m_{4}}+b,
\end{aligned}
$$

where

$$
\begin{aligned}
& a=\frac{\left(r+\lambda_{2}\right) Q_{1}+\lambda_{1} Q_{2}}{\left(r+\lambda_{1}\right)\left(r+\lambda_{2}\right)-\lambda_{1} \lambda_{2}}, \\
& b=\frac{\left(r+\lambda_{1}\right) Q_{2}+\lambda_{2} Q_{1}}{\left(r+\lambda_{1}\right)\left(r+\lambda_{2}\right)-\lambda_{1} \lambda_{2}} .
\end{aligned}
$$

For $\bar{w}_{1} \leq w<\bar{w}_{2}$, we have the equation

$$
\mathscr{L}_{2} V_{2}(w)-Q_{2}=\lambda_{2} \frac{w}{r},
$$


since $V_{1}(w)=w / r$. Thus the solution to (13) is of the form

$$
\begin{aligned}
V_{2}(w)= & C_{1} w^{n_{1}}+C_{2} w^{n_{2}}+\frac{\lambda_{2}}{r\left(r-\mu_{2}+\lambda_{2}\right)} w \\
& +\frac{Q_{2}}{r+\lambda_{2}} .
\end{aligned}
$$

Now we determine $A_{3}, A_{4}, C_{1}, C_{2}, \bar{w}_{1}$, and $\bar{w}_{2}$ using the $C^{1}$-conditions of $V_{1}$ and $V_{2}$ at $w=\bar{w}_{1}$ and $w=\bar{w}_{2}$. The $C^{1}$ condition of $V_{1}(w)$ at $w=\bar{w}_{1}$ implies

$$
\begin{aligned}
& A_{3}=\frac{(1 / r)\left(m_{4}-1\right) \bar{w}_{1}-a m_{4}}{\left(m_{4}-m_{3}\right) \bar{w}_{1}^{m_{3}},} \\
& A_{4}=\frac{(1 / r)\left(1-m_{3}\right) \bar{w}_{1}+a m_{3}}{\left(m_{4}-m_{3}\right) \bar{w}_{1}^{m_{4}} .}
\end{aligned}
$$

The $C^{1}$-condition of $V_{2}(w)$ at $w=\bar{w}_{2}$ yields

$$
\begin{aligned}
& C_{1} \\
& =\frac{\left(\left(n_{2}-1\right)\left(r-\mu_{2}\right) / r\left(r-\mu_{2}+\lambda_{2}\right)\right) \bar{w}_{2}-n_{2} Q_{2} /\left(r+\lambda_{2}\right)}{\left(n_{2}-n_{1}\right) \bar{w}_{2}^{n_{1}}}, \\
& C_{2} \\
& =\frac{\left(\left(1-n_{1}\right)\left(r-\mu_{2}\right) / r\left(r-\mu_{2}+\lambda_{2}\right)\right) \bar{w}_{2}+n_{1} Q_{2} /\left(r+\lambda_{2}\right)}{\left(n_{2}-n_{1}\right) \bar{w}_{2}^{n_{2}}} .
\end{aligned}
$$

From the $C^{1}$-condition of $V_{2}(w)$ at $w=\bar{w}_{1}$ we obtain the equations

$$
\begin{aligned}
& \frac{f_{1}\left(m_{3}\right)}{\lambda_{1}} A_{3} \bar{w}_{1}^{m_{3}}+\frac{f_{1}\left(m_{4}\right)}{\lambda_{1}} A_{4} \bar{w}_{1}^{m_{4}}+b \\
& =C_{1} \bar{w}_{1}^{n_{1}}+C_{2} \bar{w}_{1}^{n_{2}}+\frac{\lambda_{2}}{r\left(r-\mu_{2}+\lambda_{2}\right)} \bar{w}_{1}+\frac{Q_{2}}{r+\lambda_{2}}, \\
& \frac{m_{3} f_{1}\left(m_{3}\right)}{\lambda_{1}} A_{3} \bar{w}_{1}^{m_{3}}+\frac{m_{4} f_{1}\left(m_{4}\right)}{\lambda_{1}} A_{4} \bar{w}_{1}^{m_{4}} \\
& =n_{1} C_{1} \bar{w}_{1}^{n_{1}}+n_{2} C_{2} \bar{w}_{1}^{n_{2}}+\frac{\lambda_{2}}{r\left(r-\mu_{2}+\lambda_{2}\right)} \bar{w}_{1} .
\end{aligned}
$$

$$
\begin{aligned}
& \bar{w}_{2} \\
& =\frac{\left(\left(m_{3} f_{1}\left(m_{4}\right)-m_{4} f_{1}\left(m_{3}\right)\right) / \lambda_{1}\left(m_{4}-m_{3}\right)\right) a+b-\left(Q_{2} /\left(r+\lambda_{2}\right)\right)\left[\left(n_{1} \xi^{n_{2}}-n_{2} \xi^{n_{1}}\right) /\left(n_{2}-n_{1}\right)+1\right]}{\left(1 / r\left(r-\mu_{2}+\lambda_{2}\right)\right)\left[\left(r-\mu_{2}\right)\left\{\left(n_{2}-1\right) \xi^{n_{1}}+\left(1-n_{1}\right) \xi^{n_{2}}\right\} /\left(n_{2}-n_{1}\right)+\lambda_{2} \xi\right]-\left(\left(\left(m_{4}-1\right) f_{1}\left(m_{3}\right)+\left(1-m_{3}\right) f_{1}\left(m_{4}\right)\right) / r \lambda_{1}\left(m_{4}-m_{3}\right)\right) \xi} .
\end{aligned}
$$

Plugging (15) into the LHS of (17) implies

$$
\begin{aligned}
& \text { (LHS) of (17) } \\
& \begin{aligned}
= & \frac{f_{1}\left(m_{3}\right)}{\lambda_{1}} \frac{(1 / r)\left(m_{4}-1\right) \bar{w}_{1}-a m_{4}}{\left(m_{4}-m_{3}\right)} \\
& +\frac{f_{1}\left(m_{4}\right)}{\lambda_{1}} \frac{(1 / r)\left(1-m_{3}\right) \bar{w}_{1}+a m_{3}}{\left(m_{4}-m_{3}\right)}+b \\
= & \frac{\left(m_{4}-1\right) f_{1}\left(m_{3}\right)+\left(1-m_{3}\right) f_{1}\left(m_{4}\right)}{r \lambda_{1}\left(m_{4}-m_{3}\right)} \xi \bar{w}_{2} \\
& +\frac{m_{3} f_{1}\left(m_{4}\right)-m_{4} f_{1}\left(m_{3}\right)}{\lambda_{1}\left(m_{4}-m_{3}\right)} a+b,
\end{aligned}
\end{aligned}
$$

and plugging (16) into the RHS of (17) implies

(RHS) of (17)

$$
\begin{aligned}
& =\frac{\left(\left(n_{2}-1\right)\left(r-\mu_{2}\right) / r\left(r-\mu_{2}+\lambda_{2}\right)\right) \bar{w}_{2}-n_{2} Q_{2} /\left(r+\lambda_{2}\right)}{\left(n_{2}-n_{1}\right)} \\
& \cdot \xi^{n_{1}} \\
& +\frac{\left(\left(1-n_{1}\right)\left(r-\mu_{2}\right) / r\left(r-\mu_{2}+\lambda_{2}\right)\right) \bar{w}_{2}+n_{1} Q_{2} /\left(r+\lambda_{2}\right)}{\left(n_{2}-n_{1}\right)} \\
& \cdot \xi^{n_{2}}+\frac{\lambda_{2}}{r\left(r-\mu_{2}+\lambda_{2}\right)} \xi \bar{w}_{2}+\frac{Q_{2}}{r+\lambda_{2}} \\
& =\frac{1}{r\left(r-\mu_{2}+\lambda_{2}\right)}\left[\frac{\left(r-\mu_{2}\right)\left\{\left(n_{2}-1\right) \xi^{n_{1}}+\left(1-n_{1}\right) \xi^{n_{2}}\right\}}{n_{2}-n_{1}}\right. \\
& \left.+\lambda_{2} \xi\right] \bar{w}_{2}+\frac{Q_{2}}{r+\lambda_{2}}\left[\frac{n_{1} \xi^{n_{2}}-n_{2} \xi^{n_{1}}}{n_{2}-n_{1}}+1\right],
\end{aligned}
$$

From (19) and (20), we obtain
Also plugging (15) into the LHS of (18) implies

(LHS) of (18)

$$
=\frac{m_{3} f_{1}\left(m_{3}\right)}{\lambda_{1}} \frac{(1 / r)\left(m_{4}-1\right) \bar{w}_{1}-a m_{4}}{\left(m_{4}-m_{3}\right)}
$$




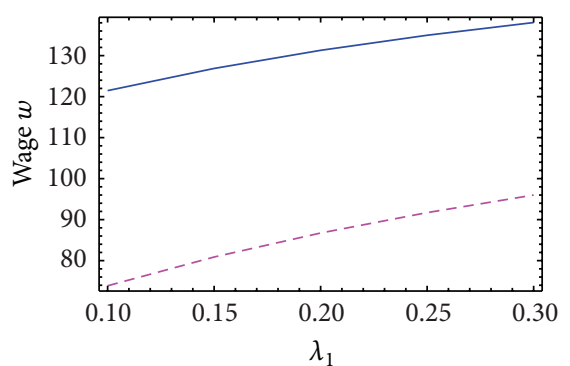

$--\bar{w}_{1}$

$-\bar{w}_{2}$

(a) Reservation wage with respect to $\lambda_{1}$ if $r=$ 0.03, $\mu_{1}=0.02, \sigma_{1}=0.2, Q_{1}=10, \mu_{2}=0.02$, $\sigma_{2}=0.4, Q_{2}=15, \lambda_{2}=0.2$

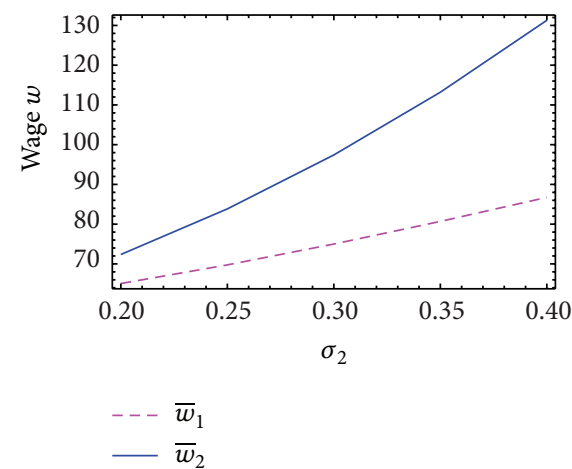

(d) Reservation wage with respect to $\sigma_{2}$ if $r=0.03$, $\mu_{1}=0.02, \sigma_{1}=0.2, Q_{1}=10, \lambda_{1}=0.2, \mu_{2}=0.02$, $Q_{2}=15, \lambda_{2}=0.2$

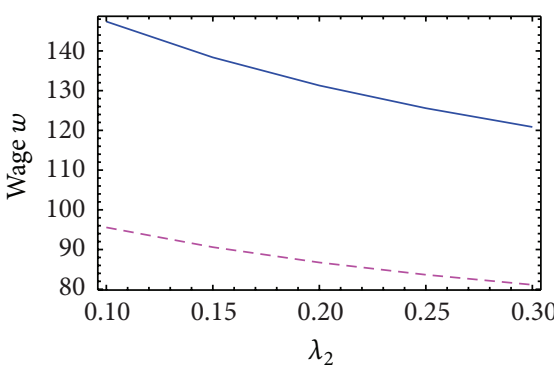

$--\bar{w}_{1}$

$-\bar{w}_{2}$

(b) Reservation wage with respect to $\lambda_{2}$ if $r=0.03$, $\mu_{1}=0.02, \sigma_{1}=0.2, Q_{1}=10, \lambda_{1}=0.2, \mu_{2}=0.02$, $\sigma_{2}=0.4, Q_{2}=15$

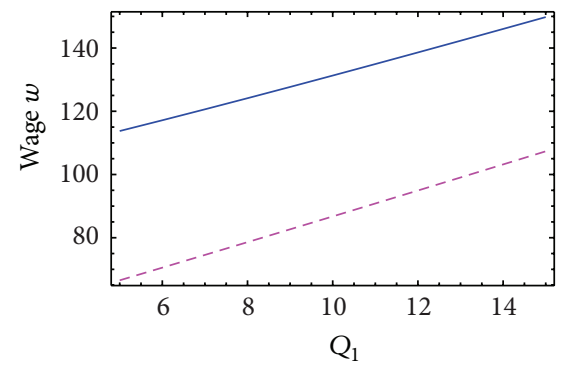

$$
---\bar{w}_{1}
$$$$
-\bar{w}_{2}
$$

(e) Reservation wage with respect to $Q_{1}$ if $r=$ $0.03, \mu_{1}=0.02, \sigma_{1}=0.2, \lambda_{1}=0.2, \mu_{2}=0.02$, $\sigma_{2}=0.4, Q_{2}=15, \lambda_{2}=0.2$

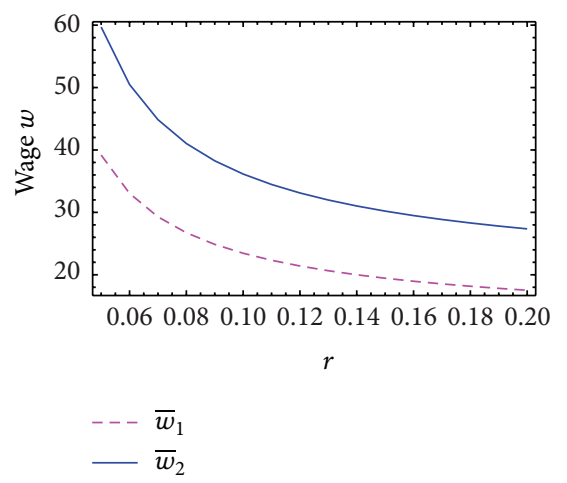

(g) Reservation wage with respect to $r$ if $\mu_{1}=$ $0.02, \sigma_{1}=0.2, Q_{1}=10, \lambda_{1}=0.2, \mu_{2}=0.02$, $\sigma_{2}=0.4, Q_{2}=15, \lambda_{2}=0.2$

FIGURE 1: Numerical implications.

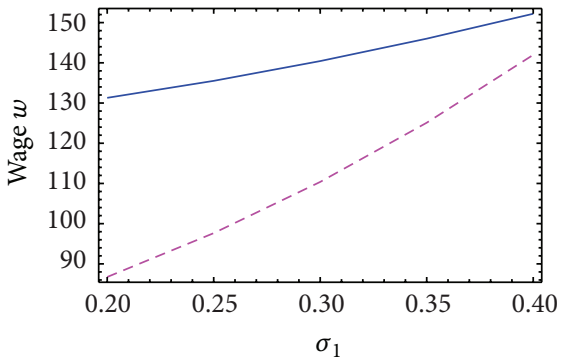

$--\bar{w}_{1}$

$-\bar{w}_{2}$

(c) Reservation wage with respect to $\sigma_{1}$ if $r=0.03$, $\mu_{1}=0.02, Q_{1}=10, \lambda_{1}=0.2, \mu_{2}=0.02, \sigma_{2}=0.4$, $\mathrm{Q}_{2}=15, \lambda_{2}=0.2$

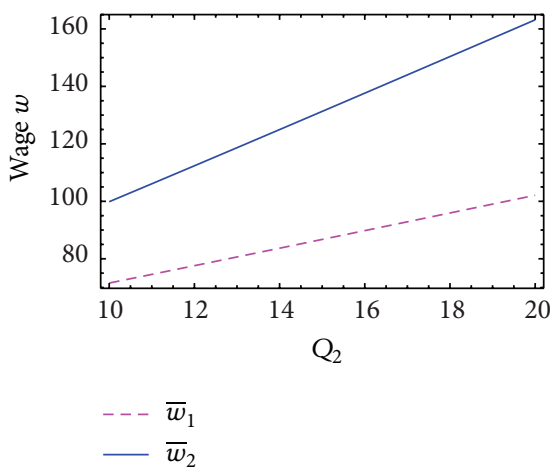

(f) Reservation wage with respect to $Q_{2}$ if $r=$ $0.03, \mu_{1}=0.02, \sigma_{1}=0.2, Q_{1}=10, \lambda_{1}=0.2$, $\mu_{2}=0.02, \sigma_{2}=0.4, \lambda_{2}=0.2$ and plugging (16) into the RHS of (18) implies

$($ RHS $)$ of $(18)=n_{1}$

$$
\begin{aligned}
& \cdot \frac{\left(\left(n_{2}-1\right)\left(r-\mu_{2}\right) / r\left(r-\mu_{2}+\lambda_{2}\right)\right) \bar{w}_{2}-n_{2} Q_{2} /\left(r+\lambda_{2}\right)}{\left(n_{2}-n_{1}\right)} \xi^{n_{1}} \\
& +n_{2} \frac{\left(\left(1-n_{1}\right)\left(r-\mu_{2}\right) / r\left(r-\mu_{2}+\lambda_{2}\right)\right) \bar{w}_{2}+n_{1} Q_{2} /\left(r+\lambda_{2}\right)}{\left(n_{2}-n_{1}\right)}
\end{aligned}
$$


with condition (21). From (23) and (24), we obtain

$$
\begin{aligned}
& \bar{w}_{2} \\
& =\frac{\left(m_{3} m_{4}\left\{f_{1}\left(m_{4}\right)-f_{1}\left(m_{3}\right)\right\} / \lambda_{1}\left(m_{4}-m_{3}\right)\right) a-\left(n_{1} n_{2} Q_{2}\left(\xi^{n_{2}}-\xi^{n_{1}}\right) /\left(r+\lambda_{2}\right)\left(n_{2}-n_{1}\right)\right)}{\left(1 / r\left(r-\mu_{2}+\lambda_{2}\right)\right)\left[\left(\left(r-\mu_{2}\right)\left\{n_{1}\left(n_{2}-1\right) \xi^{n_{1}}+n_{2}\left(1-n_{1}\right) \xi^{n_{2}}\right\} /\left(n_{2}-n_{1}\right)\right)+\lambda_{2} \xi\right]-\left(\left(m_{3}\left(m_{4}-1\right) f_{1}\left(m_{3}\right)+m_{4}\left(1-m_{3}\right) f_{1}\left(m_{4}\right)\right) / r \lambda_{1}\left(m_{4}-m_{3}\right)\right) \xi} .
\end{aligned}
$$

From (22) and (25), we derive the following algebraic equation with respect to $\xi$

$$
\begin{aligned}
& \frac{\left(\left(m_{3} f_{1}\left(m_{4}\right)-m_{4} f_{1}\left(m_{3}\right)\right) / \lambda_{1}\left(m_{4}-m_{3}\right)\right) a+b-\left(Q_{2} /\left(r+\lambda_{2}\right)\right)\left[\left(\left(n_{1} \xi^{n_{2}}-n_{2} \xi^{n_{1}}\right) /\left(n_{2}-n_{1}\right)\right)+1\right]}{\left(1 / r\left(r-\mu_{2}+\lambda_{2}\right)\right)\left[\left(\left(r-\mu_{2}\right)\left\{\left(n_{2}-1\right) \xi^{n_{1}}+\left(1-n_{1}\right) \xi^{n_{2}}\right\} /\left(n_{2}-n_{1}\right)\right)+\lambda_{2} \xi\right]-\left(\left(\left(m_{4}-1\right) f_{1}\left(m_{3}\right)+\left(1-m_{3}\right) f_{1}\left(m_{4}\right)\right) / r \lambda_{1}\left(m_{4}-m_{3}\right)\right) \xi} \\
& =\frac{\left(m_{3} m_{4}\left\{f_{1}\left(m_{4}\right)-f_{1}\left(m_{3}\right)\right\} / \lambda_{1}\left(m_{4}-m_{3}\right)\right) a-\left(n_{1} n_{2} Q_{2}\left(\xi^{n_{2}}-\xi^{n_{1}}\right) /\left(r+\lambda_{2}\right)\left(n_{2}-n_{1}\right)\right)}{\left(1 / r\left(r-\mu_{2}+\lambda_{2}\right)\right)\left[\left(\left(r-\mu_{2}\right)\left\{n_{1}\left(n_{2}-1\right) \xi^{n_{1}}+n_{2}\left(1-n_{1}\right) \xi^{n_{2}}\right\} /\left(n_{2}-n_{1}\right)\right)+\lambda_{2} \xi\right]-\left(\left(m_{3}\left(m_{4}-1\right) f_{1}\left(m_{3}\right)+m_{4}\left(1-m_{3}\right) f_{1}\left(m_{4}\right)\right) / r \lambda_{1}\left(m_{4}-m_{3}\right)\right) \xi},
\end{aligned}
$$

and if there exists a unique solution $\xi \in(0,1)$ to $(26)$, we can sequentially determine $\bar{w}_{2}, \bar{w}_{1}, A_{3}, A_{4}, C_{1}$, and $C_{2}$.

\section{Numerical Examples}

The drift term $\mu_{\Theta_{t}}$ in the wage offer dynamics in (3) is the exponential growth rate of the wage offer and can be regarded as depending on an individual's skill and ability but not on labor market conditions or macroeconomic conditions. Therefore, we set $\mu_{1}=\mu_{2}$ in our numerical examples. On the other hand, we use $\sigma_{1} \leq \sigma_{2}$ and $Q_{1} \leq Q_{2}$. That is, we assume that, in regime 2 , schooling preference (net of education costs) and wage offer volatility are higher than those in regime 1.

In Figures 1(a) and 1(b), we plot the reservation wage $\bar{w}_{i}$ against the transition intensity $\lambda_{i}$. Figures $1(\mathrm{c})$ and $1(\mathrm{~d})$ state that a higher wage offer volatility $\sigma_{i}$ yields a higher reservation wage $\bar{w}_{i}$. These results are consistent with those of Fan [1] in the sense that the wage offer volatility yields an option value to schooling. An interesting result, however, is that $\sigma_{1}$ (resp., $\sigma_{2}$ ) has an impact on $\bar{w}_{2}$ (resp., $\bar{w}_{1}$ ) and the schooling decision in a regime is dependent on the wage offer volatility in the other regime. The role of schooling preference (net of education costs) is illustrated in Figures 1(e) and 1(f). We see that more preference in education provides an individual more incentive to postpone starting work. Again, the schooling decision in a regime is dependent on schooling preference (net of education costs) in the other regime. Lastly, an individual's opportunity cost of schooling increases with the discount rate and this is explored in Figure 1(g).

\section{Conflict of Interests}

The authors declare that there is no conflict of interests regarding the publication of this paper.

\section{Acknowledgment}

Yong Hyun Shin gratefully acknowledges the support of the Basic Science Research Program through the National
Research Foundation of Korea (NRF) funded by the Ministry of Education (NRF-2013R1A1A2058027).

\section{References}

[1] C. Fan, "Schooling as a job search process," Economics Letters, vol. 41, no. 1, pp. 85-91, 1993.

[2] M. Binder, "Schooling indicators during Mexico's 'Lost decade," Economics of Education Review, vol. 18, no. 2, pp. 183-199, 1999.

[3] R. Gronau, "Information and frictional unemployment," The American Economic Review, vol. 61, pp. 290-301, 1971.

[4] A. Dixit and R. S. Pindyck, Investment Under Uncertainty, Princeton University Press, Princeton, NJ, USA, 1994.

[5] X. Guo, J. Miao, and E. Morellec, "Irreversible investment with regime shifts," Journal of Economic Theory, vol. 122, no. 1, pp. 37-59, 2005.

[6] A. Bensoussan, Z. Yan, and G. Yin, "Threshold-type policies for real options using regime-switching models," SIAM Journal on Financial Mathematics, vol. 3, no. 1, pp. 667-689, 2012. 


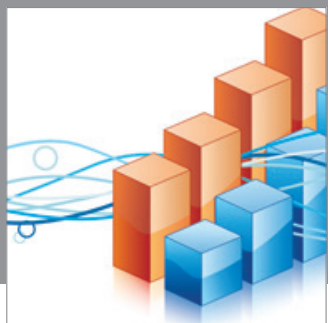

Advances in

Operations Research

mansans

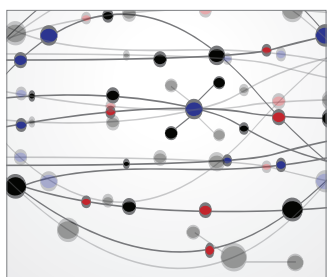

The Scientific World Journal
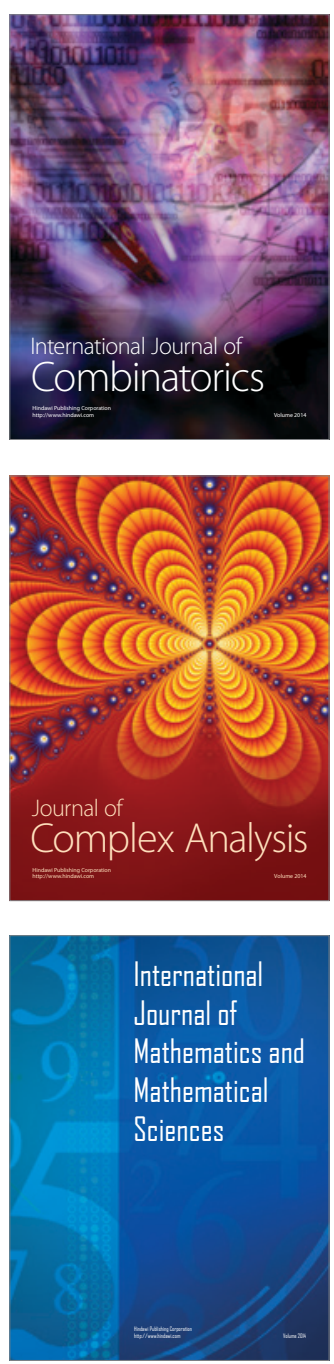
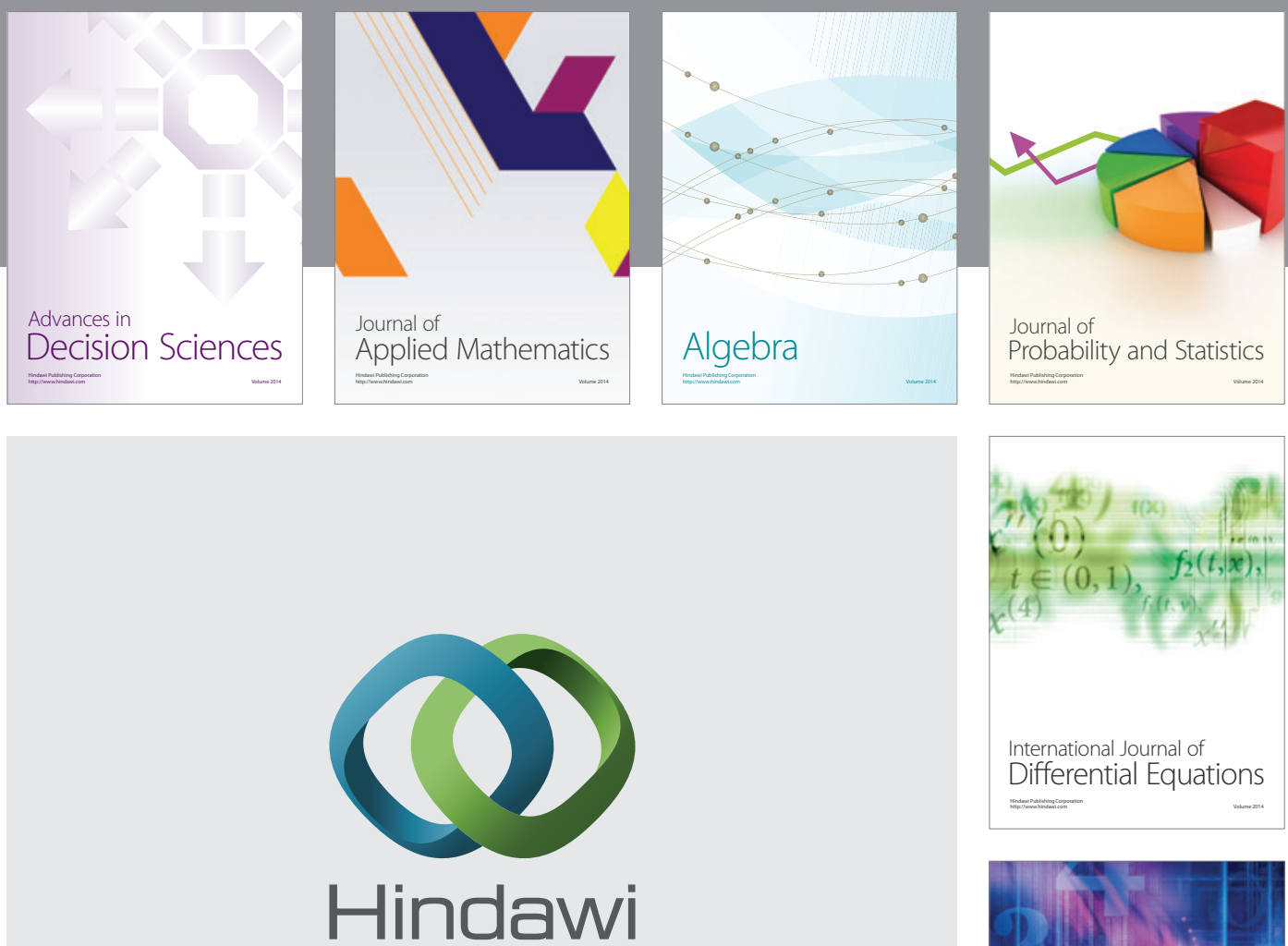

Submit your manuscripts at http://www.hindawi.com
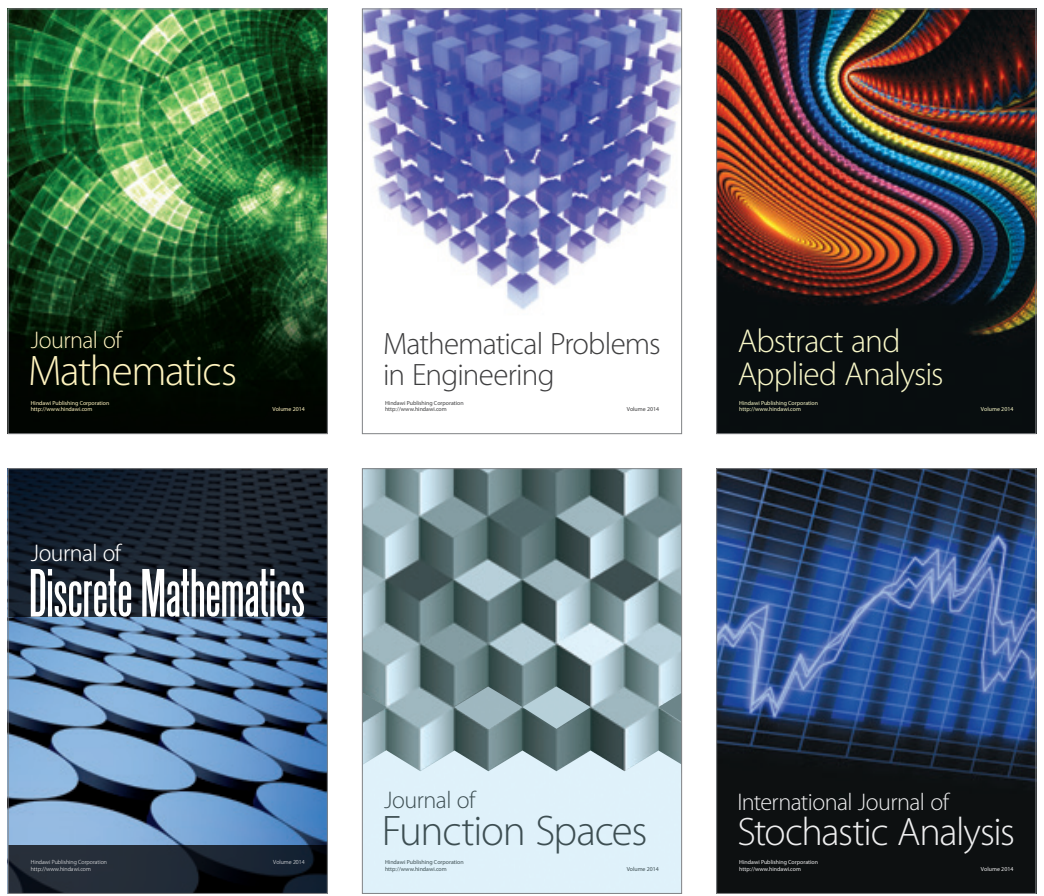

Journal of

Function Spaces

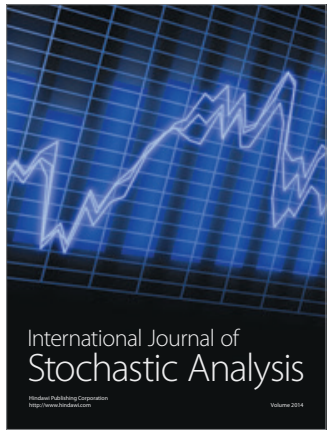

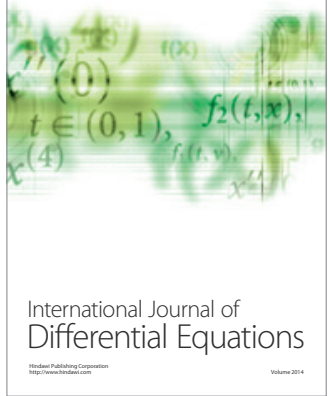
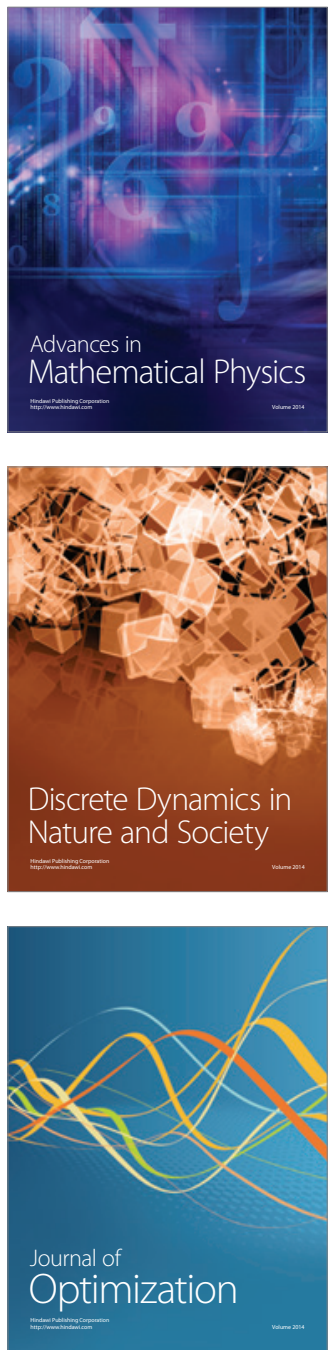